Fornace, Al

\section{Stress gene expression: analysis by infor- matics and functional genomics approaches}

\author{
Albert J. Fornace $\mathrm{Jr}^{1}$, Sally A. Amundson ${ }^{1}$, M ichael Bittner ${ }^{2}$, John \\ Weinstein ${ }^{1}$, Paul M eltzer ${ }^{2}$, Jeffrey Trent $^{2} \&$ Timothy G. M yers ${ }^{2}$ \\ ${ }^{1}$ National Cancer Institute, National Institutes of Health, \\ Bethesda, M aryland, USA \\ ${ }^{2}$ National Human Genome Research Institute, National Institutes of Health, \\ Bethesda, M aryland, USA
}

\begin{abstract}
Molecular responses to genotoxic stress are complex and are mediated by a variety of regulatory pathways. One key element in cellular response is the stress gene transcription factor $\mathrm{p} 53$, which can regulate nearly 200 genes that have already been identified. Although p53 has a central role in the cellular response to DNAdamaging agents such as ionizing radiation (IR), other pathways can also have important roles. One example is the transcriptional responses associated with IRinduced apoptosis, where induction of some genes is limited to p53 wild-type cells that also have the ability to undergo rapid apoptosis after irradiation. In contrast, other genes are triggered after IR in lines undergoing rapid apoptosis regardless of p53 status. From this and other examples, it is apparent that the pattern of stress gene expression is cell-type specific in both primary and transformed lines. The premise will be developed that such differences in stress gene responsiveness can be employed as molecular markers using a combination of informatics and functional genomics approaches. An example will be given using the panel of lines of the NCI anticancer drug screen where both the p53 status and sensitivity to a large collection of cytotoxic agents have been determined. The use of cDNA microarray hybridization to measure IR-stress gene responses has recently been demonstrated $^{1}$ and a large number of additional IR-stress genes have been identified. The basal expression and responses of some of these genes to DNA-damaging agents varied widely in cell lines from different tissues of origin and different genetic backgrounds, highlighting the importance of cellular context to genotoxic stress responses; this also highlights the need for informatics approaches to discover and prioritize hypotheses regarding the importance of particular cellular factors. The presentation will focus on the use of combining an informatics approach with functional genomics in the study of stress responses.
\end{abstract}

1. Amundson, S.A. et al. Oncogene 18, 3666-3672 (1999).

Futscher, Bernard W.

\section{Gene expression profiles associated with MDR1 expression in a doxorubicin-resis- tant human multiple myeloma cell line}

\author{
G. Watts, B. Futscher, M. Guzman, E. Epner, M. Kunkel \\ \& S. Salmon
}

Arizona Cancer Center, University of Arizona, Tucson, Arizona 85724, USA

Multiple myeloma is a monoclonal plasma cell neoplasm that usually responds to chemotherapy but subsequently becomes drug resistant. Doxorubicin is a commonly used drug for myeloma, but its use leads to the development of resistant cells that express the MDR-1 membrane pump which actively extrudes doxorubicin and other agents from the cytoplasm of resistant cells. Patients resistant to doxorubicin often show resistance to many other drugs, some of which do not appear to be extruded by the MDR-1 pump mechanism. We used cDNA microarrays to evaluate the relative expression of approximately 5,700 human genes in a human multiple myeloma cell line (8226) and its doxorubicin-resistant subline
(8226/D40). The mRNA expression profiles were performed in duplicate. As expected, the mRNA encoding the multidrug resistance gene MDR1 was markedly increased in $8226 / \mathrm{D} 40$ versus 8226 . Overall, $1 \%-2 \%$ of the 5,700 genes analysed were differentially expressed, including a variety of genes not thought to be directly related to the MDR1 mechanism. Other genes affected by doxorubicin selection included N1 spermine/spermidine acetyltransferase, CD10 and raf. These observations suggest that changes in the expression of genes other than MDR1 may participate in the emergence of the multidrug resistant phenotype. Identification of such genes provides a basis for hypothesis generation with respect to their roles in multidrug resistance.

Galbraith, David W.

\section{Implementing microarrays for the study of plant genomics}

\section{David Galbraith ${ }^{1}$, Elizabeth Pierson ${ }^{1}$, M ichael Deyholos ${ }^{1}$, Wenying $\mathrm{Xu}^{1}{ }^{1}$, George Watts ${ }^{1}$, Rene Feyereisen ${ }^{1}$, Hans Bohnert', Ken Feldmann ${ }^{1}$, Virginia Walbot ${ }^{2} \&$ Jiri Macas ${ }^{3}$}

\author{
${ }^{1}$ University of Arizona, Arizona, USA \\ ${ }^{2}$ Stanford University, California, USA \\ ${ }^{3}$ Czech Academy of Sciences, Ceske Budejovice, Czech Republic
}

Microarrays provide the means for the large-scale analysis of gene expression patterns in living organisms. My laboratory is part of three federally funded projects that are directed toward an understanding of the regulation of gene expression in higher plants.

In the first project, funded by the United States Department of Agriculture (USDA) and the Human Frontier Science Program, we are examining the functions and expression patterns of the approximately 340 members of the cytochrome P450 gene superfamily in Arabidopsis thaliana. The second project, funded by the NSF program in Plant Genomics, involves analysis of the changes in gene expression that accompany imposition of drought and salinity stress on plants. The final project, also funded by the NSF Plant Genome program, proposes to categorize at least 50,000 ESTs and cDNAs from maize (Zea mays) and provide this information to the public sector. In all of these projects, microarrays have an integral role. In the first case, we are studying tissue- and stimulus-specific gene expression to define the catalytic activities of the different P450s. In the second case, we are employing microarrays to unravel hierarchical patterns of gene regulation in response to imposed stress. For the final project, we are responsible for providing microarrays of the sequenced ESTs as a service to the maize scientific community.

We are working to solve a variety of problems at all levels, including identification of suitable substrates for printing, debugging programs for commercial arrayers, establishing sensitivity and reproducibility criteria, implementing highthroughput methods including sample tracking and methods for data analysis, reduction and archiving.

Finally, my laboratory provides campus-wide microarraying and scanning services for the University of Arizona, and we are an integral part of the core program in cancer biology currently underway at the Arizona Cancer Center. 